International Journal of Pure and Applied Mathematics

Volume 112 No. 2 2017, 369-380

ISSN: 1311-8080 (printed version); ISSN: 1314-3395 (on-line version)

url: http://www.ijpam.eu

doi: $10.12732 /$ ijpam.v112i2.14

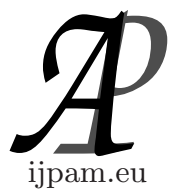

\title{
GENERALIZATION OF $\theta$-CLOSED SETS IN TOPOLOGICAL SPACES
}

\author{
P.G. Patil ${ }^{1 \S}$, S.S. Benchalli ${ }^{2}$, Tulasa Rayanagoudra ${ }^{3}$ \\ 1,2,3 Department of Mathematics \\ Karnatak University \\ Dharwad, 580 003, Karnataka, INDIA
}

\begin{abstract}
In this paper, we have introduced a new class of $\theta$-closed sets called $\theta \omega \alpha$-closed sets in topological spaces. The class of $\theta \omega \alpha$-closed sets lies between the class of $\theta$-closed and $\theta$ g-closed sets. Further, we study the some of its properties.
\end{abstract}

AMS Subject Classification: 54A05

Key Words: topological spaces, $\theta$-closed sets, $\omega \alpha$-closed sets, $\theta \omega \alpha$-closed sets, $\theta \omega \alpha$-derived set

\section{Introduction and Preliminary}

The notion of $\theta$-open sets introduced by Velicko [18] in 1968 for the purpose of studying the characterizations of H-closed Topological Spaces and these sets are stronger than the open sets. Noiri and Jafari [15], Joseph [10], Dickman and Porter [5], Long and Herrington [13] continued the work of Velicko and also they have obtained several interesting results related to these sets. The main purpose of this paper is to introduce new weaker forms of $\theta$-closed sets called $\theta \omega \alpha$-closed sets by using $\omega \alpha$-open sets of Benchalli [1]. Further, we study the properties, characterizations of $\theta \omega \alpha$-closed sets and also the relation of these sets with the existing closed sets in toplogical spaces.

Received: October 6, 2016

Revised: $\quad$ November 24, 2016

Published: $\quad$ July 16, 2017

(c) 2017 Academic Publications, Ltd. url: www.acadpubl.eu

$\S_{\text {Correspondence author }}$ 
Throughout this paper the space $(X, \tau)$ (or simply $\mathrm{X}$ ) represents the topological space on which no separation axioms are assumed unless otherwise mentioned. Let $\mathrm{A}$ be a subset of a space $(\mathrm{X}, \tau)$ then $\operatorname{cl}_{\theta}(\mathrm{A}), \operatorname{int}_{\theta}(\mathrm{A})$ and $A_{\theta}^{\mathrm{C}}$ denote the $\theta$-closure of $\mathrm{A}, \theta$-interior of $\mathrm{A}$ and $\theta$-compliment of $\mathrm{A}$ respectively. A point $\mathrm{x} \in \mathrm{X}$ is called a $\theta$-adherent point of $\mathrm{A}[18]$, if $U^{\mathrm{C}} \cap \mathrm{A} \neq \phi$ for every open set $\mathrm{U}$ of $\mathrm{X}$ containing $\mathrm{x}$. The set of all $\theta$-adherent points of $\mathrm{A}$ is called the $\theta$-closure of $\mathrm{A}$ and is denoted by $C l_{\theta}(A)$. A subset $\mathrm{A}$ of $\mathrm{X}$ is called $\theta$-closed if $\mathrm{A}=C l_{\theta} \mathrm{A}$. The complement of $\theta$-closed set is called $\theta$-open set. $\theta$-interior (denoted by $i n t_{\theta}$ ) of a subset $\mathrm{A}$ of $\mathrm{X}$ is the union of all $\theta$-open sets contained in $\mathrm{A}$.

Definition 1.1. A subset A of a topological space $\mathrm{X}$ is called:

(i) g-closed set [12] (resp. $\omega$-closed [17], $\mathrm{g}^{*} \omega \alpha$-closed set [16]) if $\operatorname{cl}(\mathrm{A}) \subseteq \mathrm{U}$ whenever $\mathrm{A} \subseteq \mathrm{U}$ and $\mathrm{U}$ is open (resp. semiopen, $\omega \alpha$-open) in $\mathrm{X}$.

(ii) $\theta$-generalized closed (briefly $\theta$ g-closed) set $[8]$ if $c l_{\theta}(\mathrm{A}) \subseteq \mathrm{U}$ whenever $\mathrm{A}$ $\subseteq \mathrm{U}$ and $\mathrm{U}$ is open in $\mathrm{X}$.

(iii) $\omega \alpha$-closed [1] (resp. g $\omega \alpha$-closed [2]) if $\alpha \mathrm{cl}(\mathrm{A}) \subseteq \mathrm{U}$ whenever $\mathrm{A} \subseteq \mathrm{U}$ and $\mathrm{U}$ is $\omega$-open (resp. $\omega \alpha$-open ) in X.

Remark 1.2. In a topological space X:

(i) [8] Every $\theta$-open set is an open.

(ii) [1] Every open set is an $\omega \alpha$-open.

Definition 1.3. A topological space $(\mathrm{X}, \tau)$ is called a $R_{1}$-space [7] if every two different points with distinct closures have disjoint neighborhoods.

Remark 1.4. [4] A topological space $(\mathrm{X}, \tau)$ is $R_{1}$-space if and only if $\mathrm{Cl}(\{$ $\mathrm{x}\})=C l_{\theta}(\{\mathrm{x}\})$ for each $\mathrm{x} \in \mathrm{X}$.

\section{2. $\theta \omega \alpha$-Closed Sets and $\theta \omega \alpha-O p e n$ Sets in Topological Spaces}

Definition 2.1. A subset A of a topological space $\mathrm{X}$ is called a $\theta \omega \alpha$-closed if $c l_{\theta}(A) \subseteq \mathrm{U}$ whenever $\mathrm{A} \subseteq \mathrm{U}$ and $\mathrm{U}$ is $\omega \alpha$-open in $\mathrm{X}$.

The complement of $\theta \omega \alpha$-closed set is called $\theta \omega \alpha$-open in X. The collection of all $\theta \omega \alpha$-closed sets and $\theta \omega \alpha$-open sets of $\mathrm{X}$ are denoted by $\theta \omega \alpha C(X)$ and $\theta \omega \alpha O(X)$

Theorem 2.2. Every $\theta$-closed set is $\theta \omega \alpha$-closed. 
Proof: Let $A$ be a $\theta$-closed and $U$ be an $\omega \alpha$-open set containing $A$ in $X$. Since $A$ is $\theta$-closed, $A=c l_{\theta}(A)$ for every subset $A$ of $X$, it implies that $c_{\theta}(A)$ $\subseteq U$. Hence $A$ is a $\theta \omega \alpha$-closed set.

Example 2.3. Let $\mathrm{X}=\{\mathrm{a}, \mathrm{b}, \mathrm{c}\}$ with $\tau=\{\phi,\{\mathrm{a}, \mathrm{b}\}, \mathrm{X}\}$. In this topological space $(X, \tau)$, a subset $A=\{b, c\}$ is $\theta \omega \alpha$-closed but not $\theta$-closed in $\mathrm{X}$.

Theorem 2.4. Every $\theta \omega \alpha$-closed set is $\theta$ g-closed but not conversly.

Proof: Let $A$ be any $\theta \omega \alpha$-closed and $U$ be an open set such that $A \subseteq U$. From Remark (ii) in 1.2 and $A$ is $\theta \omega \alpha$-closed set, it implies that $c l_{\theta}(A) \subseteq U$. Therfore $A$ is $\theta g$-closed set in $X$.

Example 2.5. Let $\mathrm{X}=\{\mathrm{a}, \mathrm{b}, \mathrm{c}\}$ and $\tau=\{\phi,\{\mathrm{a}\}, X\}$. In this topological space $(\mathrm{X}, \tau)$, the subset $\mathrm{A}=\{\mathrm{a}, \mathrm{c}\}$ is $\theta$ g-closed but not $\theta \omega \alpha$-closed set in $\mathrm{X}$.

Theorem 2.6. Every $\theta \omega \alpha$-closed set is g-closed [11] [12] and hence $\alpha g$ closed,gs-closed and rg-closed set[6].

Proof: Let $A$ be a $\theta \omega \alpha$-closed and $U$ be an open set such that $A \subseteq X$. Since $A$ is $\theta \omega \alpha$-closed set and also from remark (ii) in 1.2, it implies that $\operatorname{cl}_{\theta}(A) \subseteq$ $U$. But $c l(A) \subseteq \operatorname{cl}_{\theta}(A) \subseteq U$ [4],it implies that $c l(A) \subseteq U$. Hence $A$ is g-closed in $X$.

However the converse of the above theorem need not be true as seen from the following example.

Example 2.7. In Example 2.5, a subset $A=\{\mathrm{b}\}$ is g-closed, $\alpha$ g-closed, gs-closed and rg-closed set but not $\theta \omega \alpha$-closed in $\mathrm{X}$.

Theorem 2.8. Every $\theta \omega \alpha$-closed set is $g^{*} \omega \alpha$-closed but converse need not be true.

Proof: Let $A$ be a $\theta \omega \alpha$-closed and $U$ be an $\omega \alpha$-open set in $X$ such that $A$ $\subseteq U$. Since $A$ is a $\theta \omega \alpha$-closed, then $\operatorname{cl}_{\theta}(A) \subseteq U$. But $\operatorname{cl}(A) \subseteq \operatorname{cl}_{\theta}(A) \subseteq U$ [4], it implies that $\operatorname{cl}(A) \subseteq U$. Hence $A g^{*} \omega \alpha$-closed set. 
Remark 2.9. Every $\theta \omega \alpha$-closed set is g $\omega \alpha$-closed set but not conversly. It follows from the Theorem 2.8 and Remark 3.2 [16].

Example 2.10. Let $X=\{\mathrm{a}, \mathrm{b}, \mathrm{c}\}$ with $\tau=\{\phi,\{\mathrm{a}\},\{\mathrm{a}, \mathrm{b}\}, X\}$. In this topological space the subset $\mathrm{A}=\{\mathrm{c}\}$ is $\mathrm{g}^{*} \omega \alpha$-closed and $\mathrm{g} \omega \alpha$-closed but not $\theta \omega \alpha$-closed in $\mathrm{X}$.

Remark 2.11. The class of $\theta \omega \alpha$-closed set is independent of the class of closed, $\alpha$-closed [14], semiclosed [11] and $\omega \alpha$-closed [1]. This can be varified from following examples.

Example 2.12. Let $\mathrm{X}=\{\mathrm{a}, \mathrm{b}, \mathrm{c}\}$ with $\tau=\{\phi,\{\mathrm{a}\},\{\mathrm{a}, \mathrm{b}\}, X\}$.

In this topological space $(\mathrm{X}, \tau)$, a subset $\mathrm{A}=\{\mathrm{c}\}$ is closed, $\alpha$-closed, semiclosed and $\omega \alpha$-closed set but not $\theta \omega \alpha$-closed set.

Example 2.13. Let $(X, \tau)$ be a topological space where $X=\{a, b, c\}$ with $\tau=\{\phi,\{\mathrm{a}\},\{\mathrm{b}, \mathrm{c}\}, X\}$. The subset $\mathrm{A}=\{\mathrm{b}\}$ is $\theta \omega \alpha$-closed set but not closed, $\alpha$-closed, semiclosed and $\omega \alpha$-closed in $\mathrm{X}$.

From the above results, it follows that the $\theta \omega \alpha$-closed set is properly placed between $\theta$-closed and $\theta$ g-closed set and also we obtain the following Figure 1.

The below implications are not reversible as shown in the above examples and also from the related papers[6] and [7].

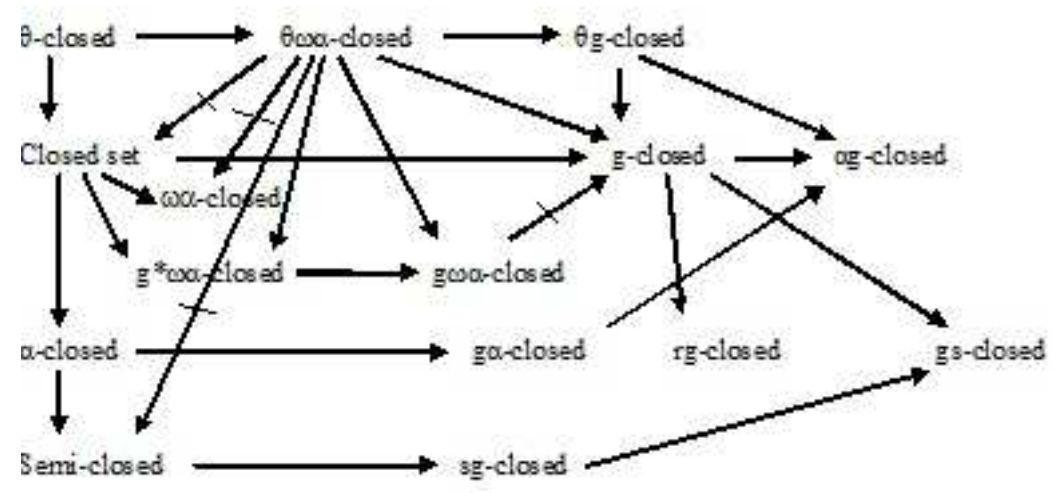

Figure 1

Theorem 2.14. Union of two $\theta \omega \alpha$-closed sets is a $\theta \omega \alpha$-closed set in $X$.

Proof: Let $A$ and $B$ be two $\theta \omega \alpha$-closed sets in $X$. Let $U$ be an $\omega \alpha$-open set in 
$X$ such that $A \cup B \subseteq U$. Then $A \subseteq U$ and $B \subseteq U$. Since $A$ and $B$ are $\theta \omega \alpha$-closed sets, $c l_{\theta}(A) \subseteq U$ and $c l_{\theta}(B) \subseteq U$. Therefore $c l_{\theta}(A) \cup \operatorname{cl}_{\theta}(B)=c l_{\theta}(A \cup B) \subseteq U$ [8]. Therefore $A \cup B$ is $\theta \omega \alpha$-closed set in $X$.

Theorem 2.15. If $A$ is a $\theta \omega \alpha$-closed set in $X$ and $A \subseteq Y \subseteq X$ then $A$ is a $\theta \omega \alpha$-closed set relative to $Y$.

Proof: Let $A \subseteq Y \cap U$ where $U$ is an $\omega \alpha$-open set in $X$.Then $A \subseteq Y$ and $A \subseteq U$. Since $A$ is $\theta \omega \alpha$-closed set in $X$, so $\operatorname{cl}_{\theta}(A) \subseteq U$ which implies that $Y \cap$ $\operatorname{cl}_{\theta}(A) \subseteq Y \cap U$.

Theorem 2.16. If $A$ is $\omega \alpha$-open and $\theta \omega \alpha$-closed set in $X$ then $A$ is $\theta$ closed in $X$.

Proof: By hypothesis $A$ is $\omega \alpha$-open and $\theta \omega \alpha$-closed in $X$ then $\operatorname{cl}_{\theta}(A) \subseteq A$. But we have $A \subseteq \operatorname{cl}_{\theta}(A)$, it implies that $\operatorname{cl}_{\theta}(A)=A$. Therefore $A$ is $\theta$-closed in $X$.

Theorem 2.17. If a subset $A$ of $X$ is $\theta \omega \alpha$-closed then $c_{\theta}(A)-A$ doesnot contain any non empty $\theta$-closed set in $X$.

Proof: Assume that $A$ is $\theta \omega \alpha$-closed set and $U$ be a non empty $\theta$-closed subset of $c_{\theta}(A)-A$.Now $A \subseteq(X-U)$ where $(X-U)$ is $\theta$-open set and also

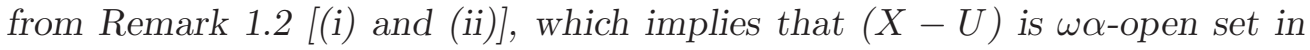
$X$. By hypothesis, $A$ is $\theta \omega \alpha$-closed, $c l_{\theta}(A) \subseteq X-c l_{\theta}(A)$ that is $U \subseteq X-c l_{\theta}(A)$. Now we have $U \subseteq c_{\theta}(A)$ and $U \subseteq X-c l_{\theta}(A)$, then $U \subseteq c l_{\theta}(A) \cap X-c l_{\theta}(A)=$ $\phi$. Therfore $U=\phi$.

The converse of the above theorem need not be true as seen in the following example.

Example 2.18. In the Example 2.13, a subset $A=\{a\}$ of $X$ then $c l_{\theta}(A)$ $\mathrm{A}=\{\mathrm{b}, \mathrm{c}\}$ doesnot contain any non empty $\theta$-closed set in $\mathrm{X}$ but $\mathrm{A}$ is not a $\theta \omega \alpha$-closed in $\mathrm{X}$.

Theorem 2.19. A subset $A$ of a space $X$ is $\theta \omega \alpha$-closed if and only if $c l(A)-A$ contains no non empty $\omega \alpha$-closed set in $X$.

Proof: Suppose $A$ is $\theta \omega \alpha$-closed in X.Let $V$ be a $\omega \alpha$-closed subset of $c l_{\theta}(A)$ $A$. Since $A$ is $\theta \omega \alpha$-closed set, $A \subseteq(X-V)$ where $(X-V)$ is an $\omega \alpha$-open set X.It 
gives that $\operatorname{cl}_{\theta}(A) \subseteq(X-V)$ then $V \subseteq X-c_{\theta}(A)$. Now we have $V \subseteq \operatorname{cl}_{\theta}(A)$ and $V$ $\subseteq X-c l_{\theta}(A)$ then $V \subseteq c l_{\theta}(A) \cap X-c l_{\theta}(A)=\phi$. Therefore $V=\phi$.

Conversely, assume that $c l_{\theta}(A)-A$ contains no non empty $\omega \alpha$-closed.Let $A \subseteq V$ where $V$ is $\omega \alpha$-open. If $c l_{\theta}(A)$ does not contain in $V$ then $c l_{\theta}(A) \cap(X-V)$ is non empty $\omega \alpha$-closed subset of $c_{\theta}(A)-A$ that is $c l_{\theta}(A) \cap(X-V) \neq \phi$ which is a contradiction to hypothesis. Therefore $A$ is a $\theta \omega \alpha$-closed set in $X$.

Theorem 2.20. A subset $A$ of a topological space $X$ is $\theta \omega \alpha$-open if and only if $V \subseteq \operatorname{int}_{\theta}(A)$ where $V$ is $\omega \alpha$-closed set in $X$.

Proof: Suppose $A$ is $\theta \omega \alpha$-open in $X$. Let $V \subseteq A$ where $V$ is $\omega \alpha$-closed set in $X$, then $(X-A) \subseteq(X-V)$. Then $c_{\theta}(X-A)=X-$ int $_{\theta}(A)[3] \subseteq(X-A)$. Therefore $V \subseteq \operatorname{int}_{\theta}(A)$.

Conversely, Assume that $V$ is $\omega \alpha$-closed set with $V \subseteq$ int $_{\theta}(A)$ whenever $V \subseteq$ $A$. Then $(X-A) \subseteq(X-V)$ and $X-\operatorname{int}_{\theta}(A)=\operatorname{cl}_{\theta}(X-A)[3] \subseteq(X-V)$. It shows that the $(X-V)$ is a $\theta \omega \alpha$-closed and subset $A$ of $X$ becomes $\theta \omega \alpha$-open in $X$.

Theorem 2.21. A subset $A$ of $X$ is $\theta \omega \alpha$-open in $X$ if and only if $U=X$ where $\operatorname{int}_{\theta}(A) \cup(X-A) \subseteq U$ and $U$ is an $\omega \alpha$-open in $X$.

Proof: Suppose a subset $A$ is an $\theta \omega \alpha$-open set in $X$. Let $U$ be an $\omega \alpha-$ open set and $\operatorname{int}_{\theta}(A) \cup(X-A) \subseteq U$.Then $(X-U) \subseteq X-\left[\operatorname{int}_{\theta}(A) \cup(X-A)\right]=(X-$ $\left.\operatorname{int}_{\theta}(A)\right)-(X-A) \Rightarrow(X-V) \subseteq \operatorname{cl}_{\theta}(X-A)-(X-A)$.Then by Theorem $2.19,(X-U)$ $=\phi$.Therefore $X=U$.

Conversely, suppose $V$ is an $\omega \alpha$-closed and $V \subseteq A$, then $(X-A) \subseteq(X-V) \Rightarrow$ $\operatorname{int}_{\theta}(A) \cup(X-A) \subseteq \operatorname{int}_{\theta}(A) \cup(X-V)$. By hypothesis, we get int ${ }_{\theta}(A) \cup(X-V)=$ $X$ and $V \subseteq \operatorname{int}_{\theta}(A)$.Hence by theorem 2.20, $A$ is $\theta \omega \alpha$-open set in $X$.

Theorem 2.22. If $A \subseteq B \subseteq X$ where $A$ is $\theta \omega \alpha$-open relative to $B$ and $B$ is $\theta \omega \alpha$-open in $X$ then $A$ is $\theta \omega \alpha$-open set in $X$.

Proof: Let $V$ be a $\omega \alpha$-closed set in $X$. Suppose $V \subseteq A$ then $V=V \cap B$ is $\omega \alpha$-closed in B. But $A$ is $\theta \omega \alpha$-open relative to $B$ and from Theorem 2.20 it implies that $V \subseteq$ int $_{\mathrm{B}_{\theta}}(A)$. Since $\operatorname{int}_{\mathrm{B}_{\theta}}(A)$ is an $\theta$-open set relative $B . V \subseteq U$ $\cap B \subseteq A$ for some $\theta$-open set $U$ in $X$.Since $B$ is a $\theta \omega \alpha$-open set in $X$. We have $V \subseteq$ int $_{\theta}(B) \subseteq B$. Therefore $V \subseteq$ int $_{\theta}(B) \cap U \subseteq B \cap U \subseteq A$. It implies that $V$ $\subseteq \operatorname{int}_{\theta}(A)$.Therefore hy Theorem 2.20, $A$ is $\theta \omega \alpha$-open set in $X$.

Theorem 2.23. In a topological space $(X, \tau), \omega \alpha O(X, \tau)=\theta C(X, \tau)$ if and only if every subset of $X$ is a $\theta \omega \alpha$-closed in $(X, \tau)$. 
Proof: Assume that every subset of $X$ is $\theta \omega \alpha$-closed.Let $U \in \omega \alpha O(X, \tau)$. Since $U \subseteq U$ and $U$ is $\theta \omega \alpha$-closed set,so $C l_{\theta}(U) \subseteq U$ then $C l_{\theta}(U)=U$ and $U \in \theta C(X, \tau)$. Therefore $\omega \alpha O(X, \tau) \subseteq \theta C(X, \tau)$. Let $V \in \theta C(X, \tau)$ then $(X$ $V)$ is $\theta$-open and hence it is $\omega \alpha$-open subset of $(X, \tau)$. Therefore $(X-V) \in$ $\omega \alpha O(X, \tau)$. Hence $\omega \alpha O(X, \tau)=\theta C(X, \tau)$

Conversely, Assume that $\omega \alpha O(X, \tau)=\theta C(X, \tau)$. Let $A$ be a subset of $X$ such that $A \subseteq U$ where $U \in \omega \alpha O(X, \tau)$, then $U \in \theta C(X, \tau)$.It implies that $c l_{\theta}(U)=$ $U$ and also $c l_{\theta}(A) \subseteq c l_{\theta}(U)=U$, then $c l_{\theta}(A) \subseteq U$.Hence $A$ is $\theta \omega \alpha$-closed set in $X$.

Theorem 2.24. Let $A$ and $B$ be any two subsets of $X$. If $A$ is a $\theta \omega \alpha$-closed and $A \subseteq B \subseteq \operatorname{cl}_{\theta}(A)$, then $B$ is $\theta \omega \alpha$-closed in $X$.

Proof: By hypothesis, $A \subseteq B$ and $B \subseteq \operatorname{cl}_{\theta}(A)$, then $\operatorname{cl}_{\theta}(B) \subseteq \operatorname{cl}_{\theta}(A)$ and $c l_{\theta}(B)-B \subseteq c l_{\theta}(A)-A$. Since $A$ is $\theta \omega \alpha$-closed set in $X$, then $c l_{\theta}(A)-A$ has no non empty $\omega \alpha$-closed set in $X$ from Theorem 2.19 and also $c_{\theta}(B)-B$ has no non empty $\omega \alpha$-closed set in X.Again by Theorem $2.19 \mathrm{~B}$ is a $\theta \omega \alpha$-closed set in X.

Theorem 2.25. Let $A$ and $B$ be any two subsets of $X$. If $\operatorname{int}_{\theta}(A) \subseteq B \subseteq$ $A$ and $A$ is $\theta \omega \alpha$-open in $X$ then $B$ is an $\theta \omega \alpha$-open set in $X$.

Proof: By hypothesis, $\operatorname{int}_{\theta}(A) \subseteq(B) \subseteq A$ then $(X-A) \subseteq(X-B) \subseteq X-$ $\operatorname{int}_{\theta}(A)=\operatorname{cl}_{\theta}(X-A)$. Since $A$ is $\theta \omega \alpha$-closed set, then $(X-A)$ is $\theta \omega \alpha$-closed set. From Theorem 2.24, $(X-B)$ is $\theta \omega \alpha$-closed set in $X$. Hence $B$ is $\theta \omega \alpha$-open set in $X$.

Definition 2.26. We recall that a topological space $X$ is said to be a regular space if $\forall$ closed subset $F$ of $X$ and for each point $x \in X$ and $x \notin F$ there exist disjoint open sets $\mathrm{G}, \mathrm{H}$ of $\mathrm{X}$ such that $\mathrm{F} \subset \mathrm{G}$ and $\mathrm{x} \in \mathrm{H}$.

Theorem 2.27. A subset $A$ of a regular space $X$ is $g^{*} \omega \alpha$-closed then $A$ is $\theta \omega \alpha$-closed set.

Proof: Since the space $X$ is regular, then $\operatorname{cl}_{\theta}(A)=c l(A)$ [3]. By hypothesis, $A$ is $g^{*} \omega \alpha$-closed set,it implies that $\operatorname{cl}_{\theta}(A)=c l(A) \subseteq U$ whenever $A \subseteq U$ and $U$ is $\omega \alpha$-open set. Hence $A$ is $\theta \omega \alpha$-closed in $X$.

Theorem 2.28. Let $X$ be a $R_{1}$-space then for a singleton subset $A$ of $X$ the following results are equivalent: 
(i) $A$ is $\theta \omega \alpha$-closed

(ii) $A$ is $g^{*} \omega \alpha$-closed.

Proof: (i) $\Rightarrow$ (ii) Follows from the Theorem 2.8 .

(ii) $\Rightarrow$ (i).From Remark 1.4, the concepts of $\theta$-closure and closure are coincide for singleton sets. Then replace $c_{\theta}(A)$ by $c l(A)$ in the Theorem 3.8.

\section{3. $\theta \omega \alpha$-Closure and $\theta \omega \alpha$-Interior in Topological Spaces}

In this section, we introduce the notion of $\theta \omega \alpha$-Closure and $\theta \omega \alpha$-Interior of topological spaces.

Definition 3.1. The $\theta \omega \alpha$-closure of a subset $\mathrm{A}$ of $\mathrm{X}$ is denoted by $\theta \omega \alpha c l(A)$ and is defined as the intersection of all $\theta \omega \alpha$-closed sets containing A.

Therefore $\theta \omega \alpha c l(A)=\cap\{\mathrm{F} \subseteq \mathrm{X}: \mathrm{A} \subseteq \mathrm{F}$ and $\mathrm{F}$ is $\theta \omega \alpha$-closed set $\}$.

Definition 3.2. The $\theta \omega \alpha$-interior of a subset $\mathrm{A}$ of $\mathrm{X}$ is denoted by $\theta \omega \alpha \operatorname{int}(A)$ and is defined as the union of all $\theta \omega \alpha$-open sets contained in $\mathrm{A}$. Therefore $\theta \omega \alpha \operatorname{int}(A)=\cup\{\mathrm{G} \subseteq \mathrm{X}: \mathrm{G} \subseteq \mathrm{A}$ and $\mathrm{G}$ is $\theta \omega \alpha$-open set $\}$.

Remark 3.3. If $\mathrm{A} \subseteq \mathrm{X}$ then,

(i) $\mathrm{A} \subseteq \theta \omega \alpha c l(A) \subseteq \operatorname{cl}_{\theta}(\mathrm{A})$.

(ii) $\operatorname{int}_{\theta}(\mathrm{A}) \subseteq \theta \omega \alpha \operatorname{int}(A) \subseteq \mathrm{A}$.

Theorem 3.4. Let $A$ and $B$ be any two subsets of a space $X$ then the following properties are true

(i) $A$ is $\theta \omega \alpha$-closed if and only if $\theta \omega \alpha c l(A)=A$.

(ii) $A$ is $\theta \omega \alpha$-closed set in $X$ then $\theta \omega \alpha c l(A)$ is the smallest $\theta \omega \alpha$-closed subset of $X$ containing $A$.

(iii) $\theta \omega \alpha c l(\phi)=\phi$ and $\theta \omega \alpha c l(X)=X$

(iv) $\theta \omega \alpha c l(A)$ is a $\theta \omega \alpha$-closed set in $X$.

(v) If $A \subseteq B$, then $\theta \omega \alpha c l(A) \subseteq \theta \omega \alpha c l(B)$

(vi) $\theta \omega \alpha c l(A \cup B)=\theta \omega \alpha c l(A) \cup \theta \omega \alpha c l(B)$

(vii) $\theta \omega \alpha c l(A \cap B) \subseteq \theta \omega \alpha c l(A) \cap \theta \omega \alpha c l(B)$

$(\operatorname{viii}) \theta \omega \alpha c l(\theta \omega \alpha c l(A))=\theta \omega \alpha c l(A)$ 
Proof: (i) Let $A$ be a $\theta \omega \alpha$-closed set in $X$. Since $A \subseteq A$ and $A \in\{F \subseteq X$ : $A \subseteq F$ and $F$ is $\theta \omega \alpha$-closed set $\}$ it implies that $A=\cap\{F \subseteq X: A \subseteq F$ and $F$ is, $\theta \omega \alpha$-closed set $\} \subseteq A$.Then $\theta \omega \alpha c l(A) \subseteq A$.But $A \subseteq \theta \omega \alpha c l(A)$ is always true.Hence $A=\theta \omega \alpha c l(A)$

Conversely, suppose $A=\theta \omega \alpha c l(A)$,then $\theta \omega \alpha c l(A)$ is $\theta \omega \alpha$-closed in $X$ which implies that $A$ is $\theta \omega \alpha$-closed set in space $X$.

(ii) Suppose $A$ is $\theta \omega \alpha$-closed set in $X$ then $\theta \omega \alpha c l(A)=\cap\{F \subseteq X$ : $A \subseteq F$ and $F$ is, $\theta \omega \alpha$-closed $\}$. Since $A \subseteq A$ and $A$ is $\theta \omega \alpha$-closed in $X$, from $i^{s} t, A=\theta \omega \alpha c l(A)$ is the smallest $\theta \omega \alpha$-closed set in $X$ containing $A$.

(iii) and (iv) - Follows from the Definition 3.1

(v) We know that $B \subseteq \theta \omega \alpha c l(B)$, then $A \subseteq B \subseteq \subseteq \theta \omega \alpha c l(B)$. So $\theta \omega \alpha c l(B)$ is the smallest $\theta \omega \alpha$-closed set containing $A . \rightarrow($ a).$B u t \theta \omega \alpha c l(A)$ is the smallest $\theta \omega \alpha$-closed set containing $A . \rightarrow(b)$ From (a) and (b) we get, $\theta \omega \alpha c l(A)$ is smaller than $\theta \omega \alpha \operatorname{cl}(B)$.Therfore $\theta \omega \alpha c l(A) \subseteq \theta \omega \alpha c l(B)$

(vi) We know that $A \subseteq A \cup B$ and $B \subseteq A \cup B$,from (v), $\theta \omega \alpha c l(A) \subseteq \theta \omega \alpha \operatorname{cl}(A$ $\cup B)$ and $\theta \omega \alpha c l(B) \subseteq \theta \omega \alpha \operatorname{cl}(A \cup B)$. Then $\theta \omega \alpha c l(A) \cup \theta \omega \alpha c l(B) \subseteq \theta \omega \alpha \operatorname{cl}(A$ $\cup B) \rightarrow(a)$. Let $x \in X$ be any point such that $x \notin \theta \omega \alpha c l(A) \cup \theta \omega \alpha c l(B)$, then there exist $\theta \omega \alpha$-closed sets $G$ and $H$ such that $A \subseteq G$ and $B \subseteq H, x \notin G$ and $x \notin H$, it implies that $x \notin G \cup H$ and $A \cup B \subseteq G \cup H$. By the Theorem $2.14 G \cup H$ is $\theta \omega \alpha$-closed, then $\mathrm{x} \notin \theta \omega \alpha \operatorname{cl}(A \cup B)$. Therefore $\theta \omega \alpha \operatorname{cl}(A \cup B)$ $\subseteq \theta \omega \alpha c l(A) \cup \theta \omega \alpha c l(B) \rightarrow(b)$. From (a) and (b) we have, $\theta \omega \alpha \operatorname{cl}(A \cup B)=$ $\theta \omega \alpha c l(A) \cup \theta \omega \alpha c l(B)$.

(vii) We know that $A \cap B \subseteq A$ and $A \cap B \subseteq B$. From (v) we have, $\theta \omega \alpha \operatorname{cl}(A$ $\cap B) \subseteq \theta \omega \alpha c l(A)$ and $\theta \omega \alpha \operatorname{cl}(A \cap B) \subseteq \theta \omega \alpha c l(B)$. Then $\theta \omega \alpha \operatorname{cl}(A \cap B) \subseteq$ $\theta \omega \alpha c l(A) \cap \theta \omega \alpha c l(B)$.

(viii) From Definition 3.1 we have, $\theta \omega \alpha c l(A)$ is a $\theta \omega \alpha$-closed set in $X$. Let $\theta \omega \alpha c l(A)=G$, then $G$ is a $\theta \omega \alpha$-closed set in $X$. From (i) we have, $\theta \omega \alpha c l(G)=$ G. It implies that $\theta \omega \alpha c l(\theta \omega \alpha c l(A))=\theta \omega \alpha c l(A)$.

The converse of property (ii) and property (vii) are need not be true as seen in the following examples.

Example 3.5. Let $X=\{a, b, c\}$ with $\tau=\{\phi,\{a\},\{b\},\{a, b\}, X\}$. In this topological space, a subset $\mathrm{A}=\{\mathrm{a}\}$, then $\theta \omega \alpha c l(A)=\{\mathrm{a}, \mathrm{c}\}$ which is the smallest $\theta \omega \alpha$-closed set in $\mathrm{X}$ containing $\mathrm{A}$ but $\mathrm{A}$ is not a $\theta \omega \alpha$-closed in $\mathrm{X}$.

Example 3.6. In the Example 3.5, subsets $A=\{a\}$ and $B=\{b\}$, then $\theta \omega \alpha c l(A)=\{\mathrm{a}, \mathrm{c}\}, \theta \omega \alpha c l(B)=\{\mathrm{b}, \mathrm{c}\}$ and $\theta \omega \alpha \operatorname{cl}(\mathrm{A} \cap \mathrm{B})=\phi$. Therefore $\{\mathrm{c}\}=\theta \omega \alpha \mathrm{cl}(\mathrm{A}) \cap \theta \omega \alpha \operatorname{cl}(\mathrm{B}) \nsubseteq \theta \omega \alpha \mathrm{cl}(\mathrm{A} \cap \mathrm{B})=\phi$.

Definition 3.7. [9] Let A be any subset of a space X. Then $\omega \alpha$-kernel of 
$\mathrm{A}$ is denoted by $\omega \alpha-\operatorname{ker}(\mathrm{A})$ and is defined as intersection of all $\omega \alpha$-open sets containing A. Then $\omega \alpha-\operatorname{ker}(\mathrm{A})=\cap\{\mathrm{U} \subseteq \mathrm{X}: \mathrm{A} \subseteq \mathrm{U}$ and $\mathrm{U}$ is $\omega \alpha$-open set $\}$

Theorem 3.8. A subset $A$ of a space $X$ is $\theta \omega \alpha$-closed if and only if $c l_{\theta}(A)$ $\subseteq \omega \alpha-\operatorname{ker}(A)$.

Proof: Let $U$ be an $\omega \alpha$-open set containing $A$ then $\operatorname{cl}_{\theta}(A) \subseteq \omega \alpha-k e r(A) \subseteq$ $U$.Therefore $A$ is $\theta \omega \alpha$-closed set.

Conversely, suppose $A$ is $\theta \omega \alpha$-closed in $X$. Then $\operatorname{cl}_{\theta}(A) \subseteq U$ whenever $A \subseteq U$ and $U$ is $\omega \alpha$-open in $X$. Let $\mathrm{x} \in \operatorname{cl}_{\theta}(A)$. If $\mathrm{x} \notin \omega \alpha-k e r(A)$ then there exists an $\omega \alpha$-open set $U$ containing $A$ such that $\mathrm{x} \notin U$.Then $\mathrm{x} \notin c l_{\theta}(A)$, which is a contradiction to the hypothesis.Hence $c l_{\theta}(A) \subseteq \omega \alpha-\operatorname{ker}(A)$.

Definition 3.9. Let $\mathrm{N}$ be any subset of topological space $\mathrm{X}$ then $\mathrm{N}$ is said to be $\theta \omega \alpha$-neighborhood (denoted by $\theta \omega \alpha$-nbd ) of point $\mathrm{x} \in \mathrm{X}$ if there exist an $\theta \omega \alpha$-open set $\mathrm{U}$ such that $\mathrm{x} \in \mathrm{U} \subseteq \mathrm{N}$.

Theorem 3.10. A subset $A$ of topological space $X$ is $\theta \omega \alpha$-closed and $x \in$ $\theta \omega \alpha c l(A)$ if and only if $N \cap A \neq \phi$ for any $\theta \omega \alpha-n b d N$ of $\mathrm{x}$ in $X$.

Proof: If $x \notin \theta \omega \alpha c l(A)$. Then there exists $\theta \omega \alpha$-closed set $F$ of $X$ such that $A \subseteq F$ and $\mathrm{x} \notin F$. Thus $\mathrm{x} \in(X-F)$ and $(X-F)$ is $\theta \omega \alpha$-open in $X$. But $A \cap(X$ $-F)=\phi$ which is a contradicton. Hence $\mathrm{x} \in \theta \omega \alpha \mathrm{cl}(\mathrm{A})$.

Conversely, suppose that there exists an $\theta \omega \alpha-n b d N$ of a point $x \in X$ such that $N \cap A=\phi$. Then there exists an $\theta \omega \alpha$-open set $F$ of $X$ such that $x \in F \subseteq N$. Therefore we have $F \cap A=\phi$ and $x \in(X-F)$. Then $\theta \omega \alpha c l(A) \in(X-F)$ and $x \notin$ $\theta \omega \alpha c l(A)$, which is a contradiction to hypothesis that $\mathrm{x} \in \theta \omega \alpha c l(A)$. Therefore $N \cap A \neq \phi$

Remark 3.11. The intersection of any two members of $\theta \omega \alpha \mathrm{N}(\mathrm{x})$ is again a member of $\theta \omega \alpha \mathrm{N}(\mathrm{x})$.

Definition 3.12. Let A be a subset of topological space X.Then a point $\mathrm{x} \in \mathrm{X}$ is said to be a $\theta \omega \alpha$-limit point of $\mathrm{A}$ if every $\theta \omega \alpha$-open set of $\mathrm{x}$ contains a point of $\mathrm{A}$ other than $\mathrm{x}$, that is $(\mathrm{G}-\{\mathrm{x}\}) \cap \mathrm{A} \neq \phi[$ or $G \cap(\mathrm{A}-\{\mathrm{x}\}) \neq \phi]$ for every $\theta \omega \alpha$-open set $\mathrm{G}$ of $\mathrm{x}$.

In a topological space $\mathrm{X}$, the set of all $\theta \omega \alpha$-limit points of a given subset $\mathrm{A}$ of $\mathrm{X}$ is called $\theta \omega \alpha$-derived set of $\mathrm{A}$ and is denoted by $\theta \omega \alpha \mathrm{d}(\mathrm{A})$.

Theorem 3.13. Let $A$ and $B$ be any two subsets of a space $X$ then the 
following properties are true:

(i) $\theta \omega \alpha d(\phi)=\phi$

(ii) If $A \subseteq B$, then $\theta \omega \alpha d(A) \subseteq \theta \omega \alpha d(B)$

(iii) $\theta \omega \alpha d(A \cup B)=\theta \omega \alpha d(A) \cup \theta \omega \alpha c l(B)$

(iv) $\theta \omega \alpha d(A \cap B) \subseteq \theta \omega \alpha d(A) \cap \theta \omega \alpha d(B)$.

Proof: (i) Let $\mathrm{x} \in X$ and $\mathrm{x} \in \theta \omega \alpha d(\phi)$. Then for every $\theta \omega \alpha$-open set $G$ containing $x$, we should have $[G-\{x\}] \cap A \neq \phi$, which is impossible. Therfore $\theta \omega \alpha d(\phi)=\phi$.

(ii) Let $x \in \theta \omega \alpha d(A)$ then $x$ is a limit point of $A$. From definition 3.12, $[G-\{$ $x\}] \cap A \neq \phi$ for every $\theta \omega \alpha$-nbd $G$ containing $x$. Since $A \subseteq B$, then $[G-\{x\}]$ $\cap A \subseteq[G-\{x\}] \cap B$. Therefore $x \in \theta \omega \alpha d(B)$. Hence $\theta \omega \alpha d(A) \subseteq \theta \omega \alpha d(B)$.

(iii) Since $A \subseteq A \cup B$ and $B \subseteq A \cup B$ then from property (ii), $\theta \omega \alpha d(A)$ $\cup \theta \omega \alpha d(B) \subseteq \theta \omega \alpha d(A \cup B) \rightarrow(a)$. On the other hand if $\mathrm{x} \notin \theta \omega \alpha d(A) \cup$ $\theta \omega \alpha d(B)$, then $x \notin \theta \omega \alpha d(A)$ and $x \notin \theta \omega \alpha d(B)$. Therefore there exist $\theta \omega \alpha-n b d s$ $G_{1}$ and $G_{2}$ of $x$ such that $G_{1} \cap(A-\{x\})=\phi$ and $G_{2} \cap(B-\{x\})=\phi$. Since $G_{1} \cap G_{2}$ is $\theta \omega \alpha-n b d$ of $x$, then we get $\left(G_{1} \cap G_{2}\right) \cap[A-\{x\}]=\phi$. Therefore $x$ notin $\theta \omega \alpha d(A \cup B)$. Therfore $\theta \omega \alpha d(A \cup B) \subseteq \theta \omega \alpha d(A) \cup \theta \omega \alpha d(B)-\rightarrow(b)$. Therefore from (a) and (b) we get, $\theta \omega \alpha d(A \cup B)=\theta \omega \alpha d(A) \cup \theta \omega \alpha c l(B)$. (iv)- Since $A \cap B \subseteq A$ and $A \cap B \subseteq B$, then from property (ii) we have $\theta \omega \alpha d(A$ $\cup B) \subseteq \theta \omega \alpha d(A)$ and $\theta \omega \alpha d(A \cap B) \subseteq \theta \omega \alpha d(B)$. Consequently, $\theta \omega \alpha d(A \cap B) \subseteq$ $\theta \omega \alpha d(A) \cap \theta \omega \alpha d(B)$

\section{Acknowledgments}

The authors are grateful to the University Grant Commission, New Delhi, India for its financial support under UGC-SAP-III DRS to the Department of Mathematics, Karnatak University, Dharwad, India. Under F.510/3/DRSIII/2016(SAP-I) dated: 29-02-2016. Also this research work is supported by the National Fellowship for students of OBC by University Grants Commission, New Delhi, India. Under F./2015-16/NFO-2015-17-OBC-KAR-31351/ (SA-III/ Website) dated:01-04-2015.

\section{References}

[1] S. S. Benchalli, P. G. Patil and T. D. Rayanagaudar, $\omega \alpha$-Closed Sets in Topological Spaces, The Global J. Appl. Math. and Math. Sci., 2, No. 1-2 (2009), 53-63.

[2] S. S. Benchalli, P. G. Patil and P. M. Nalwad, Generalized $\omega \alpha$-Closed Sets in Topological Spaces, J. New Results in Science, 7 (2014), 7-19. 
[3] M. Caldas, S. Jafari and M. M. Kovar, Some Properties of $\theta$-open Sets, Divulg. Mat., 12 No. 2 (2004), 161-169.

[4] J. Cao, M. Ganster, Reilly and M. Steiner, $\delta$-closure, $\theta$-closure and Generalized Closed Sets, Appl. Gen. Topology, Univesidad Polotecnica de Valencia, 6, No. 1 (2005),79-89.

[5] R. F. Dickman Jr. and J. R. Porter, A-closed Subsets of Hausdorff Spaces, Pacific J. Math., 59, No. 2 (1975), 407-415.

[6] J. Dontchev, On Generalizing Semi-Preopen Sets, Mem. Fac. Sci. Kochi Univ. Ser. A Math., 16 (1995), 35-48.

[7] J. Dontchev and M. Ganster, On $\delta$-generalised Closed Sets and $T_{3 / 4}$-Spaces, Mem. Fac.Sci. Kochi Univ. Ser. A Math., 17 (1996), 15-31.

[8] J. Dontchev and H. Maki, On $\theta$-generalised Closed Sets, Int. J. Math. and Math. Sci., 22, No. 2 (1999), 239-249.

[9] S. Jafari, S. S. Benchalli, P. G. Patil and T. D.Rayanagoudar, Pre $\mathrm{g}^{*}$-Closed Sets in Topological Spaces, J. of Advaced Studies in topology, 3, No. 3 (2012), 55-59.

[10] J. E. Joseph, $\theta$-Closure and $\theta$-Subclosed Graphs, Math. Chronicle, 8 (1979), 99-117.

[11] N. Levine, Semi-open Sets and Semi-continuity in Topological Spaces, Amer. Math.Monthly, 70, No. 1 (1963), 36-41.

[12] N. Levine, Generalised Closed Sets in Topology, Rend. Circ. Mat. Palermo, 19, No. 2 (1970), 89-96.

[13] P. E. Long and L.L. Herrington, The $T_{\theta}$-Topology and Faintly Continuous Functions, Kyungpook Math. J., 22 (1982), 7-14.

[14] O. Njastad, On Some Classes of Nearly Open Sets, Pacific. J. Math., 15, No. 3 (1965), 961-970.

[15] T. Noiri and S. Jafari, Properties of $(\theta, \mathrm{S})$-continuous Functions, Topology and its Applications, 123, No. 1 (2002), 167-179.

[16] P. G. Patil, S. S. Benchalli and Pallavi S. Mirajakar, Generalized star $\omega \alpha$-closed Sets in Topological Spaces, J. New Results in Science, 9 (2015), 37-45.

[17] P. Sundaram and M.Sheik John, On $\omega$-closed Sets in Topology, Acta Ciencia Indica Mathematics, 26, No. 4 (2000), 389-392.

[18] N. V. Velicko, H-Closed Topological Spaces, Amer. Math. Soc. Transl., 78, No. 2 (1968), 103-118. 